\title{
Evaluation of the effect of chamomile extract on lingual histological changes in rabbit received 5-fluorouracil (histological and immunohistochemical study)
}

Accepted: 17/4/2016

\begin{abstract}
Shukria Muhammed Zahawi * $\quad$ Ali Sultan Al-Refai ** Hanan Abdulla Abdulqader $* * *$
Abstract

Background and objective: Chamomile has been used as an herbal medication since ancient times and is still popular until today. This study aimed to investigate the potential anticytotoxic effect of chamomile extract on the lingual mucosa of rabbits treated with 5-fluorouracil (5-FU).

Methods: Thirty six rabbits were used in the study. For induction of tongue mucositis for the study group, $5-\mathrm{FU}$ at a dose of $(4 \mathrm{mg} / \mathrm{kg})$ was administered intraperitoneally one time daily for five successive days. The control animals were intraperitoneally injected by normal saline one time daily for five successive days. The rats in each group were randomly divided into two groups: Distilled water and chamomile extract treated groups (9 animals each). A volume of distilled water equal to chamomile extract was given by gavage tube, while the other group was gavaged with chamomile extract at a dose of (100 $\mathrm{mg} / \mathrm{kg}$ ) one time daily. The treatment with distilled water or the chamomile extract was continued for sixteen days and then the animals were sacrificed, and the tongues were excised for histopathological and immunohistochemical analysis.

Results: The 5-FU/water treated group showed a significant increase in histological damage scores, decrease in the ki-67and increase in caspase -3 epithelial immune expression $(P<0.05)$ in comparison with saline/water treated group. The 5-FU/chamomile treated group showed a significant increase in damage score, significant decrease in ki-67 immune expression and significant increase in caspase-3 immune expression $(P<0.05)$ in comparison with 5-FU/water treated group.

Conclusion: Chamomile extract at a dose of $(100 \mathrm{mg} / \mathrm{kg})$ one time daily for sixteen days cannot be used for the treatment of oral mucositis. It causes damage to the tissue, decreases proliferation, and increases apoptosis.
\end{abstract}

Keywords: Lingual papillae; Chamomile; 5-FU; Ki-67; Caspase- 3.

\section{Introduction}

Most treatments used to kill cancer cells also kill a diverse range of normal cell types, leading to a broad range of adverse side effects like mucositis. Mucositis is inflammation of the mucous membrane, and it is commonly seen in radiation therapy as well as a patient receiving chemotherapy, the incidence varies according to the type of cancer and treatment modality. ${ }^{1}$ It affect about 40 $80 \%$ of patients treated with chemotherapy and almost all those receiving radiotherapy of the head and neck. ${ }^{2}$ 5-Flurouracil in general used for treatment of malignant tumors, particularly of the breast, rectum or colon and in the treatment of uterine, primary hepatic, pancreatic, gastric, ovarian, and bladder carcinomas. The 5-FU is one of the most common causes of oral mucositis ${ }^{4}$, and due to its structure, 5-FU interferes with nucleoside metabolism and can be integrated into DNA and RNA, leading to cytotoxicity and cell death. ${ }^{3}$ Oral mucositis may results in discontinuation of chemotherapy or unplanned treatment. ${ }^{4-6}$ One of the widely used herbs for medicinal purposes is

* Department of Oral Diagnosis, College of Dentistry, Hawler Medical University, Erbil, Iraq.

** Department of Oral and Maxillofacial Surgery, College of Dentistry, Hawler Medical University, Erbil, Iraq.

*** Department of Oral Diagnosis, College of Dentistry, Duhok University, Duhok, Iraq. 
chamomile whose standardized tea and herbal extracts are prepared from dried flowers of Matricaria species. It is a well-known widely used plant for various gastro-intestinal disorders, and widely used for many human ailments such as hay fever, muscle spasms, inflammation, menstrual disorders, insomnia, ulcers, rheumatic pain, and hemorrhoids. ${ }^{7}$ Chamomile has antioxidant, antiinflammatory, antibacterial and antifungal properties. ${ }^{8}$ The majority of their antioxidant activity is due to flavones, falvenols, isoflavones, anthocyanin, cumarin, tannic acid, and isocatechins. ${ }^{9}$ Chamomile extracts were shown to cause less growth inhibitory effects on normal cells, but showed significant reductions in cell viability in various human cancer cell lines. Srivastava and Gupta found that chamomile exposure induced apoptosis in cancer cells but not in normal cells at similar doses. ${ }^{10}$ The tongue exhibits a lining or rather a continuous mucosa of different thicknesses, throughout its surface. The epithelium is thick and tough on the dorsal surface where tongue wear is greater due to friction with food. ${ }^{11}$ The ultra structural elements of the epithelium vary in the morphology of different mammals and show several types of papillae. Besides being associated with animal species, variations may be also related to type of food and to the animal's adaptation to environmental conditions. ${ }^{12}$ The epithelium of the dorsum surface of the tongue is consist of four types of lingual papillae, namely, filiform, foliate, fungiform, and vallate papillae, featuring mechanical and taste functions. ${ }^{13}$ However, shape, size, and organization of the tongue papillae change according to each mammal species. ${ }^{14} \mathrm{Ki}-67$ antigene is one of different cell-cycle regulating proteins which can be determined by immunohistochemistry. ${ }^{15}$ The Ki-67 antibody reacts with a nuclear non-histone protein of $395 \mathrm{KD}$ that is express in all active phases of the cell cycle, except the G0. Ki-67 is encoded by the MKI67 gene and is an excellent marker to determine the growth fraction of a given cell population. ${ }^{16}$ Caspase- 3 , encoded by the CASP3 gene, is a member of the cysteine-aspartic acid protease family. Stimulation of caspase- 3 is a key indicator of apoptosis. ${ }^{17}$ Many chemotherapeutic agents target caspase- 3 in order to promote apoptosis, these drugs induce apoptosis by molecular mechanisms mediated through mitochondrial dysfunction. ${ }^{18,19}$ The current study was aimed to investigate the preventive role of chamomile extract (100 mg/kg/day) on the lingual mucosa of male rabbits treated with 5-fluorouracil. To evaluate the grade of damage or protection, we used histological and immunohistochemical investigations to clarify its effect on cell proliferation and apoptosis.

\section{Methods}

Thirty six healthy adult male rabbits (Oryctolagus cuniculus) were used in the study. They were around four months old, and weighting $(1.2-1.8) \mathrm{kg}$. All rabbits were housed in the animal house at the College of Medicine, Hawler Medical University, Erbil, Kurdistan Region of Iraq, under similar laboratory conditions in a temperature-controlled environment $\left(21-24^{\circ} \mathrm{C}\right)$, with a $12 \mathrm{hr}$ light $/ 12 \mathrm{hr}$ dark cycle and free access to food and water, and allowed to acclimatize for 7 days before use. The research project was approved by the Research Ethics Committee at College of Dentistry, Hawler Medical University under the protocol. The rabbits were randomly divided by simple random allocation into two groups (18 rabbits each):

Control groups (intraperitoneally injected by physiological saline):

Consisted of distilled water treated group and chamomile extract treated group (9 animals each), and the treatment continued for 16 days. In the distilled water treated group, a volume of distilled water equal to chamomile extract was given by gavage needle, while the chamomile 
extract treated group was gavaged with chamomile extract (Matricaria chamomilla organic alcoholic extract-United States-Code HS3751002) at a dose of $100 \mathrm{mg} /$ day administered orally one time daily. ${ }^{20}$ A physiological saline $(0.9 \% \mathrm{NaCl})$ in a similar dose of $5-\mathrm{FU}(4 \mathrm{mg} / \mathrm{kg})$ administered intraperitoneally one time daily for five successive days to all animals in this group.

\section{Study groups (intraperitoneally injected} by $5-\mathrm{FU})$ :

Consisted also of distilled water treated group and chamomile extract treated group (9 animals each), and the treatment continued for 16 days. They were gavaged by distilled water or chamomile extract in a similar way as the control groups. Intraperitoneal injections of 5-FU at a dose of $(4 \mathrm{mg} / \mathrm{kg})$ was administered one time daily for five successive days for all animals in this group. This standard dose is well-tolerated and showing minimal weight loss. $^{21,22}$ At the end of 16 days, animals were sacrificed by ketamine over dose. Tongues were carefully excised, longitudinally bisected and immediately fixed in neutral buffered $10 \%$ formalin, processed for hematoxylin and eosin staining (H\&E), and for immunohistochemical analysis using $\mathrm{Ki}-67$ and cleaved caspase-3 immunolabeling.

\section{Hematoxylin and eosin analysis:}

Analysis for tongue damage scores (mucositis scores for study groups) were carried out among the groups. Five regions in the epithelial area and five regions in the connective tissue were selected (linearly adjacent to each other) and preceded with scoring the damage of these separate locations. The changes were assessed by one blind evaluator to the type of the sample with scores of 0 to 5 . The semi quantitative scores used were as follows; Grade $0=$ Normal, Grade 1 $($ minimal $)=<5 \%$, Grade $2($ mild $)=6-20 \%$, Grade 3 (moderate) $=21-50 \%$, Grade 4 (marked) $=51-75 \%$, and Grade 5(severe) $=76-100 \%$. This method was modified from the method proposed by Üçuncu et al. to assess the degree of tongue mucositis. ${ }^{23}$

The damaged areas were evaluated for the following:

${ }^{*}$ Cell changes in stratified squamous epithelium such as hyperchromasia, pleomorphism, binucleation, and necrosis.

* Degeneration and vacuolar alteration of the basal layer.

* Congestion of blood vessels.

* Inflammatory infiltrate in the submucosa.

Immunohistochemical analysis:

Cell proliferation was assessed by $\mathrm{Ki}-67$ immunohistochemistry, while the apoptosis was assessed by cleaved caspase-3 immunostaining. They were performed by using monoclonal Mouse Anti-Human Ki-67 Antigen, Clone MIB-1, Code No. M 7240 staining system, and a monoclonal Mouse Anti-Human Caspase-3 Oncoprotein Clone 124 Code No 1587 ready to use $\mathrm{N}$ - series primary antibody, for use with DakoEnVision TM, EnVision TM double staining and LASAB TM 2 systems. The staining procedures followed the instructions included with each detection system. Positive and negative controls were run simultaneously with the biopsy specimen. Positive cells expressing Ki-67 were identified by the brown nucleus, while cleaved caspase-3 was demonstrated brown cytoplasmic and nuclear staining. To ensure the objectivity of the analysis, the evaluation was carried out by two independent observers. Five sections were randomly chosen for each animal. Approximately 1000 epithelial cells were counted by two observers at a magnification of $400 \mathrm{x}$ and the percentages of Ki-67 and caspase- 3 positive cells were calculated. All microscopic analyses were performed using a light microscope (Olympus, Tokyo, Japan). The levels of $\mathrm{Ki}-67$ and caspase -3 expressions were evaluated according to the scoring system of Seleit et al.: [(absent: $<1 \%$ ), (mild: 1-10\%), (moderate: >10-50\%), (strong: $>50 \%$ )]. ${ }^{24}$

Statistical analysis: After necessary data collection, the results were given as mean \pm standard deviation. Statistical 


\section{Results}

Hematoxylin and eosin results:

Saline/ Water treated group (Figure 1; A1, A2, A3): The dorsal surface of the tongue was normal. The filiform papillae were numerous, evenly distributed, thin, with a pointed tip. They were regular in size, shape and orientation, with a normal covering keratinized epithelium. The fungiform papillae were also revealed normal covering epithelium; they were few in numbers, short, broad, vascular, and scattered between the filiform papillae. The muscular core consists of a mass of skeletal muscle of longitudinal, transverse and oblique fibers.

Saline/Chamomile treated group (Figure 1; B1, B2, B3): The epithelium showed vacuolation and hyperchromasia. The connective tissue shows mild congestion and inflammatory cells infiltration.
5-FU/Water treated group: (Figure 1; C1, C2, C3): Lingual papillae of tongue reveled loss of the normal appearance of filiform papillae, and most of them showed a flattening of the tips with the loss of their characteristic conical shape, and the fungiform papillae also showed atrophic changes. Epithelial vacuolation, congestion of blood vessels with different types of inflammatory cells infiltration in the connective tissue were also seen.

5-FU/Chamomile treated group (Figure 1; D1, D2): Loss of the saw tooth appearance of filliform papillae, severe vacuolar degeneration of the basal layer of epithelial cells, severe inflammatory infiltrate and congestion of blood vessels were also seen. The statistical analysis regarding damage scores in all studied groups is seen in Table 1.

Table 1: Comparison between groups in the means \pm standard deviations of the histopathological damage scores, Ki-67 and Caspase- 3 immune expression used in the study in the dorsal tongue mucosa following water or chamomile administration in rabbits post saline or 5-FU injection.

\begin{tabular}{lcccccc}
\hline Groups & $\begin{array}{c}\text { Damage } \\
\text { score }\end{array}$ & $\boldsymbol{P}$ value & Ki-67 & $\boldsymbol{P}$ value & Caspase-3 & $\boldsymbol{P}$ value \\
\hline Saline/water & $0.25 \pm 0.09$ & 0.0120 & $19.12 \pm 0.25$ & 0.0120 & $0.19 \pm 0.08$ & 0.250 \\
Saline/chamomile & $0.96 \pm 0.21$ & & $16.32 \pm 0.60$ & & $0.67 \pm 0.62$ & \\
& & & & & & \\
Saline/water & $0.25 \pm 0.09$ & 0.0120 & $19.12 \pm 0.25$ & 0.0120 & $0.19 \pm 0.08$ & 0.0120 \\
5-FU/water & $2.72 \pm 0.35$ & & $9.17 \pm 1.05$ & & $3.87 \pm 0.20$ & \\
& & & & & & \\
Saline/water & $0.25 \pm 0.09$ & 0.0120 & $19.12 \pm 0.25$ & 0.0120 & $0.19 \pm 0.08$ & 0.0120 \\
5-FU/chamomile & $3.70 \pm 0.32$ & & $6.18 \pm 0.69$ & & $7.27 \pm 0.43$ & \\
& & & & & & \\
Saline/chamomile & $0.96 \pm 0.21$ & 0.0120 & $16.32 \pm 0.60$ & 0.0120 & $0.67 \pm 0.62$ & 0.0120 \\
5-FU/water & $2.72 \pm 0.35$ & & $9.17 \pm 1.05$ & & $3.87 \pm 0.20$ & \\
& & & & & & \\
Saline/chamomile & $0.96 \pm 0.21$ & 0.0120 & $16.32 \pm 0.60$ & 0.0120 & $0.67 \pm 0.62$ & 0.0120 \\
5-FU/chamomile & $3.70 \pm 0.32$ & & $6.18 \pm 0.69$ & & $7.27 \pm 0.43$ & \\
& & & & & & \\
5-FU/water & $2.72 \pm 0.35$ & 0.0120 & $9.17 \pm 1.05$ & 0.0120 & $3.87 \pm 0.20$ & 0.0120 \\
5-FU/chamomile & $3.70 \pm 0.32$ & & $6.18 \pm 0.69$ & & $7.27 \pm 0.43$ & \\
\hline \hline
\end{tabular}


A1
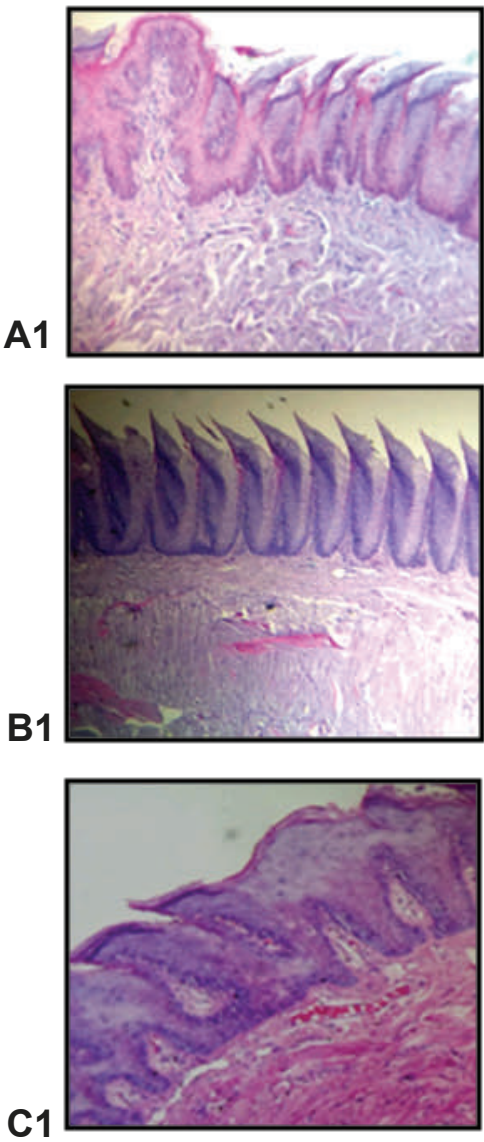

A2
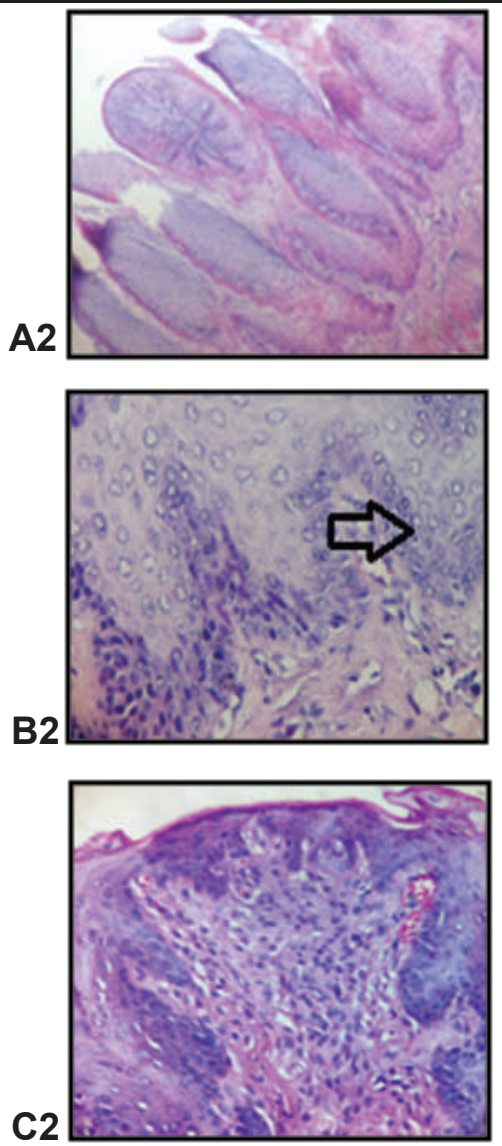
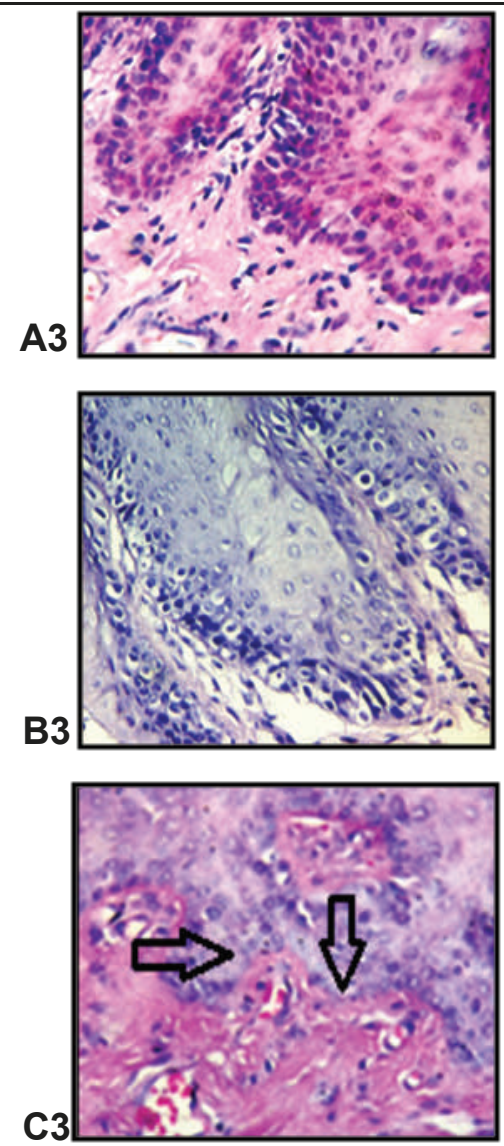
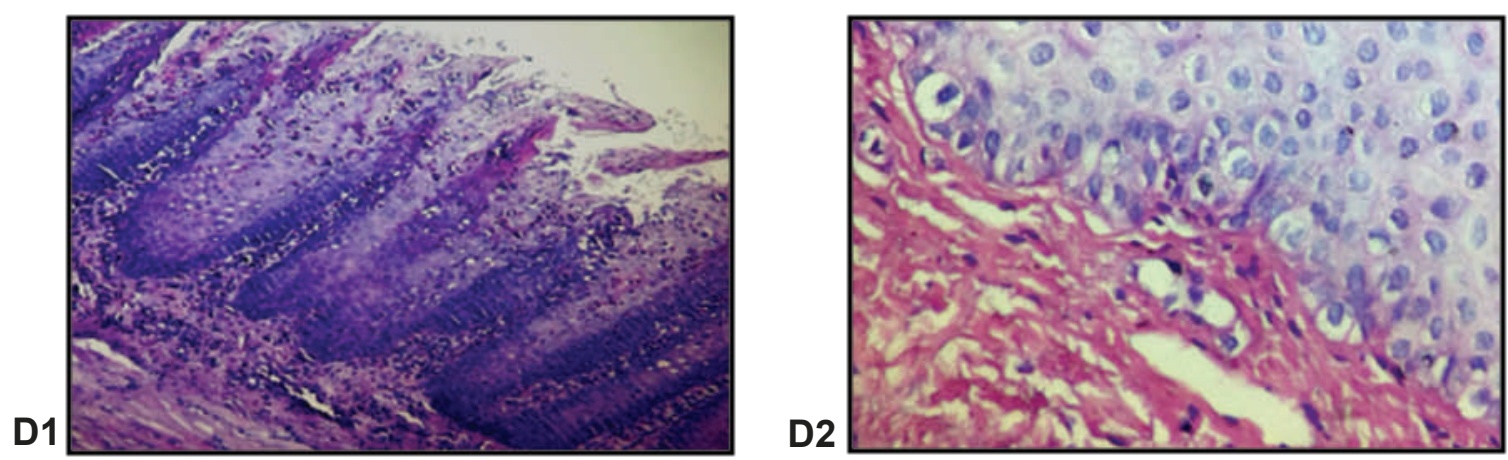

Figure 1: Photomicrograph of tongue in the saline/distilled water group showing different types of papillae, the sharp conical projections of regular filiform papillae appear with pointed tips. The basal cell layer appears normal and columnar in shape (A1, H\&Ex40; A2,H\&E×100;A3,H\&Ex400). Saline/chamomile group shows normal shape papillae with pointed tip, but the basal and supra basal cell layer appear with vacuolation (arrow), hyperchromasia and pleomorphism (B1,H\&E×100;B2,H\&E×400; B3, H\&E x 400). 5-FU/ distilled water group showing loss of filliform papillae tips, the presence of inflammatory infiltrate and congested blood vessels in the connective tissue with degeneration of basal cells layer, arrows (C1, H \& E ×100; C2, H \& E x400; C3,H\&Ex400). 5-FU/chamomile extract treated group shows loss of the saw tooth appearance of filliform papillae, severe inflammatory infiltrate and, severe degeneration changes in basal epithelial cells (D1, H\&E×100; D2, H\&E×400). 
Immunohistochemical results:

The descriptive data and their statistical analysis regarding $\mathrm{ki}-67$, and caspase- 3 immune expression in all groups are seen in Table 1 and Figure 2. The result showed that taking chamomile extract for a longe duration of time (16 days) cause a significant increase in damage score, significant decrease in ki-67 immune expression, and a nonsignificant increase in caspase- 3 immune expression in comparison with saline/water treated
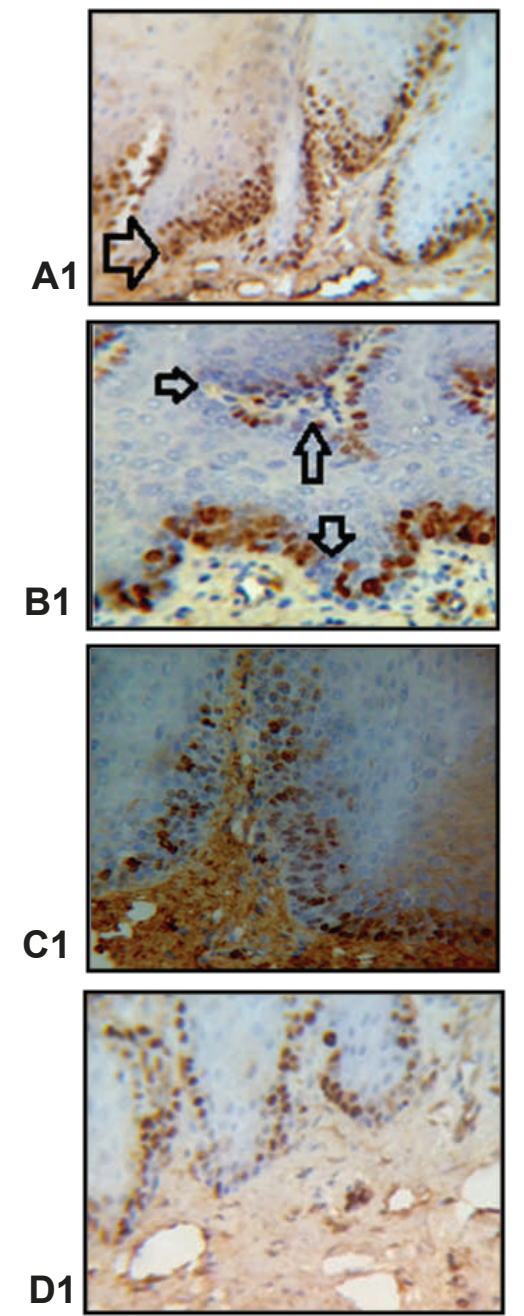

group. 5-FU cause a significant increase in damage score, significant decrease in ki-67 immune expression, and a significant increase in caspase-3 immune expression in comparison with saline/water treated group. The 5-FU/chamomile group showed a significant increase in damage score, a significant decrease in ki-67 immune expression and a significant increase in caspase-3 immune expression in comparison with 5-FU/water treated group.

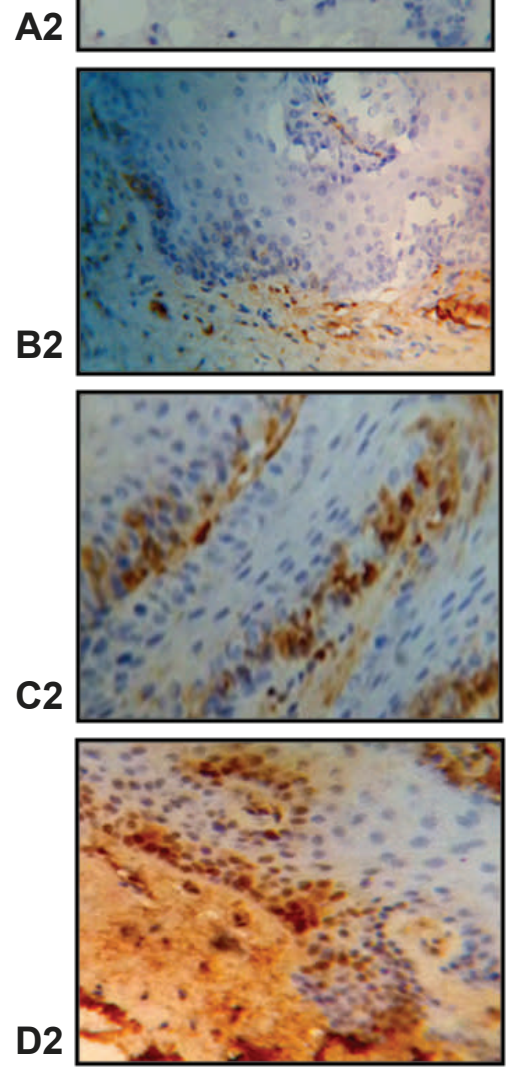

Figure 2: Photomicrograph of immunohistochemical staining results ( $x 400)$. The saline/ water treated group revealed moderate Ki-67 immuno reactivity which appears in basal and supra basal layer ( $\mathrm{A} 1$, arrow), and negative caspase- 3 immuno reactivity in epithelial cells (A2). Saline/chamomile group revealed moderate Ki-67 immuno reactivity which is missing in some areas of basal layer (B1, arrows) and negative caspase- 3 immuno reactivity in epithelial cells (B2). 5-FU /water group revealed mild Ki-67 (C1) and mild caspase-3 immuno reactivity in epithelial cells (C2). 5-FU/chamomile group revealed mild Ki-67 (D1) and mild caspase-3 immuno reactivity which appears in basal and supra basal layer (D2). 


\section{Discussion}

The oral cavity has been considered as a mirror that reflects the health of the individual. Changes indicative of disease are seen as metamorphosis in the oral mucosa lining the mouth, which can reveal systemic conditions. ${ }^{25}$ Modern anticancer treatment represents a significant challenge to the integrity of the oral mucosa. Chemotherapeutic agents and radiation therapy restricted the proliferative ability of the epithelium so that it becomes thin or ulcerated. This is manifest first in the more rapidly proliferating tissues, such as oral lining mucosa causing oral mucositis. ${ }^{26}$ 5 -FU is mostly indicated for the treatment of malignant tumors. The use of $5-\mathrm{FU}$ is one of the most common causes of oral mucositis. ${ }^{27,28}$ In the present study, the 5-FU/water treated group showed a significant increase in histological damage scores $(P<0.05)$ in comparison with saline/water treated group, this is due to the increases in the release of proinflammatory cytokines which cause tissue damage and inflammatory response resulting in increased subepithelial vascularity. ${ }^{29,30}$ In addition, the 5-FU administration was accompanied by a significant reduction in $\mathrm{Ki}-67$ positive cells and a significant increase in caspase -3 positive cells. Wright et al. found that 5-FU administration results in decreased cellularity and increased apoptosis in the small intestine. ${ }^{31}$ Mucositis appears to stem from a series of dynamic interactions as well as molecular and cellular events that involve all elements of the mucosa. 5-FU inhibits thymidylate synthase and both RNA and DNA synthesis, causing a decrease in proliferation in addition to marked apoptosis. $^{32,33}$ The result also showed that taking chamomile extract $(100 \mathrm{mg} /$ day administered orally for 16 days) cause a significant increase in damage score, a significant decrease in $\mathrm{Ki}-67$ immunoexpression and a non significant increase in caspase-3 immunoexpression in comparison with saline/water treated group. These results were surprising, as previous studies had shown normal tissue to be insensitive to chamomile extract, and chamomile extract can be used for the treatment of the different condition. ${ }^{7,8}$ The differences in the results can be due to the type and concentration of chamomile used in the study, and the duration of intake. The 5-FU/chamomile group showed a significant increase in damage score, a significant decrease in ki-67 immune expression and a significant increase in caspase-3 immune expression in comparison with 5-FU/water treated group. This indicates that chamomile extract can not be used for the treatment of oral mucositis. Studies on the effect of chamomile on oral mucosa lesions especially mucositis are scarce. Topical chamomile mouth wash was used in few studies and the results were controversial. ${ }^{34,35} \mathrm{Al}$-Refai used $100 \mathrm{mg} / \mathrm{kg}$ chamomile extract, two times daily for treatment of intestinal mucositis caused by $5-\mathrm{FU}$, and found that when chamomile extract was taken for three days, it increased the intestinal proliferation and antiapoptosis, but when it was taken for seven days, it can cause cytotoxic and damaging effect to the jejunum. ${ }^{36}$ The harmful effect of chamomile may come from its constituents, like bisabolol, volatile oils, anthemic and tannic acid, and chamazulene. Zadeh et al. and Cavalieri et al. found that $\alpha$ - bisabolol is a small oily sesquiterpene alcohol, and it is a pro-apoptotic agent and enhance apoptosis. $^{37,38}$

\section{Conclusion}

Chemotherapy like 5-fluorouracil has a deleterious effect on the oral mucosa leading to marked microscopic changes. The long duration of taking chamomile can cause a cytotoxic and damaging effect to tongue mucosa. Chamomiles do not protect the tongue mucosa from fluorouracil-induced cytotoxicity. Further studies are recommended using different concentrations of chamomile extract for a longer duration of time. 


\section{Conflicts of interest}

The authors report no conflicts of interest.

\section{References}

1. Ghom AG, Jedhe SM. Text book of oral pathology. $2^{\text {nd }}$ ed. New Delhi; Jaypee Brothers Medical Publishers; 2013.

2. Da Cruz Campos MI, Campos CN, Aarestrup FM, Aarestrup JV. Oral mucositis in cancer treatment: Natural history, prevention and treatment (review). Mol clin oncol 2014; 2(3):337-40.

3. Noordhuis P, Holwerda U, Van der Wilt CL, Van Groeningen CJ, Smid K, Meijer S, et al. 5-Fluorouracil incorporation into RNA and DNA in relation to thymidylate synthase inhibition of human colorectal cancers. Ann Oncol 2004; 15:1025-32.

4. Mead GM. Management of oral mucositis associated with cancer chemotherapy. Lancet 2002; 359(23):815-6.

5. Chen $P$, Lingen M, Sonis ST, Walsh-Reitz MM, Toback FG. Role of AMP-18 in oral mucositis. Oral Oncol 2011; 47(9):831-9.

6. Miller MM, Donald DV, Hagemann TM. Prevention and treatment of oral mucositis in children with cancer. J Pediatr Pharmacol Ther 2012; 17(4):340 -50 .

7. Srivastava JK, Eswar-Shankar E, Gupta S. Chamomile: A herbal medicine of the past with bright future. Mol Med Rep 2010; 3(6):895-901.

8. Al- Bahtiti NH. Chemical analysis and biological activity of Jordanian chamomile extracts. Adv J Food Sci Technol 2012; 4(1):22-5.

9. Svenningsen AB, Madsen KD, Liljefors $T$, Stafford GI, Van Staden J, Jäger AK. Biflavones from Rhus species with affinity for the GABA (A) benzodiazepine receptor. J Ethno pharmacol 2006; 103(2):276-80.

10. Srivastava JK, Gupta S. Antiproliferative and apoptotic effects of chamomile extract in various human cancer cells. J Agric Food Chem 2007; 55:9470-8.

11. Ferreira RJ, Carvalho AE, Souza W, Alvarenga FB, Rodrigues FB. Anatomia da Artéria Lingual Profunda em Sus scrofa domestica, LINNAEUS, 1758. Ciência Animal Brasileira 2011; 12(2): 298-305.

12. Miyawaki Y,Yoshimura K, Shindo J, Kageyama I. Light and scanning electron microscopic study on the tongue and lingual papillae of the common raccoon, Procyon lotor. Okajimas Folia Anatomica Japonica 2010; 87(2):65-73.

13. Fonseca ET, Oliveira CM, Franciolli ALR, Miglino MA. "Caracteristicas das papilas do dorso da lingua de cabras (Capra hircus): estudo por de microscopia eletrônica de varredura e luz. Pesquisa Veterinária Brasileira 2011; 31(1): 67-73.

14. Zheng J, Kobayashi K. "Comparative morphological study on the lingual papillae and their connective tissue cores (CTC) in reeves' muntjac deer (Muntiacus reevesi)," Ann Anat 2006; 188(6):555-64.

15. Cooper LS, Gillett CE, Smith P, Fentiman IS, Barnes DM. Cell proliferation measured by MIB1 and timing of surgery for breast cancer. $\mathrm{Br} J$ Cancer 1998; 77:1502-7.

16. Ermiah E, Buhmeida A, Abdalla F, Khalid BR,Salem N, Pyrhonen S. Prognostic Value of Proliferation Markers: Immunohistochemical Ki-67 Expression and Cytometric S-Phase Fraction of Women with Breast Cancer in Libya. J Cancer 2012; 3:421-31.

17. Green DR, Kroemer G. The pathophysiology of mitochondrial cell death. Science 2004; 305: 626-9.

18. Decker $\mathrm{P}$, Muller S. Modulating poly (ADP-ribose) polymerase activity: potential for the prevention and therapy of pathogenic situations involving DNA damage and oxidative stress. Curr Pharm Biotechnol 2002; 3:275-83.

19. Degterev A, Boyce M, Yuan J. A decade of caspases. Oncogene 2003; 22:8543-67.

20. Emam MAL. Comparative evaluation of antidiabetic activity of Rosmarinus officinalis and Chamomile recutita in streptozotocin induced diabetic rats. Agric Biol J N Am 2012; 3:247-52.

21. Ozel O, Aycicek A, Kenar F, Aktepe F, Sargin R, Deniz $M$, et al. Histopathologic changes in the rabbit submandibular gland after5-fluorouracil chemotherapy. Turk J Med Sci 2010; 40:213-20.

22. Barakat SA. The possible role of green tea in protecting submandibular salivary gland function in rats receiving 5-Fluorouracil 2014. M.Sc. Thesis. Mansoura University, Egypt.

23. Ücuncu H, Ertekin MV, Yoruk Ö, Sezen O, Özkan A, Erdoğan $F$, et al. Vitamin $E$ and L-carnitine, separately or in combination, in the prevention of radiation-induced oral mucositis and myelosuppression: A controlled study in a rat model. J Radiat Res 2006; 47:91-102.

24. Seleit IA, Asaad N, Maree A, Abdel Wahed M. Immunohistochemical expression of p53 and Ki-67 in cutaneous lupus erythematosus. J Egypt Women Dermatol Soc 2010; 7(1):5-15.

25. Christopher AS, Mary JK: Biology of oral mucosa and esophagus. J Natl Cancer Inst Monogr 2001; 29:7-15.

26. Zheng WK, Inokuchi A, Yamamoto T, Komiyama $S$. Taste dysfunction in irradiated patients with head and neck cancer. Fukuoka Igaku Zasshi 2002; 93(4):64-76.

27. Alvariño-Martín C, Sarrión-Pérez MG Prevention and treatment of oral mucositis in patients receiving chemotherapy. J Clin Exp Dent 2014; 6(1):74-80.

28. López BC. Oral toxicity produced by chemotherapy: A systematic review. J Clin Exp Dent 2014; 6(1):81-90.

29. Sonis ST. Mucositis as a biological process: a new hypothesis for the development of 
chemotherapy-induced stomatotoxicity. Oral Oncol 1998; 34(1):39-43.

30. Sonis ST. A biological approach to mucositis. J Support Oncol 2004; 2(1):21-32.

31. Wright TH, Yazbeck R, Lymn KA, Whitford EJ, Cheah KY, Butler RN, et al. The herbal extract, lberogast, improves jejunal integrity in rats with 5-Fluorouracil (5-FU)-induced mucositis. Cancer Biol Ther 2009; 8:923-9.

32. Pritchard DM, Watson AJ, Potten CS, Jackman AL, Hickman JA. Inhibition by uridine but not thymidine of p53 dependent intestinal apoptosis initiated by 5-fluorouracil: Evidence for the involvement of RNA perturbation. Proc Nat Acad Sci 1997; 94(5):1795-9.

33. Green DR, Kroemer G. The pathophysiology of mitochondrial cell death. Science 2004; 305: 626-9.

34. Carl W, Emrich LS. Management of oral mucositis during local radiation and systemic chemotherapy: a study of 98 patients. J Prosthet Dent 1991; 66:361-9.

35. Fidler P, Loprinzi CL, O'Fallon JR, Leitch JM, Lee $\mathrm{JK}$, Hayes DL, et al. Prospective evaluation of chamomile mouth wash for prevention of $5-\mathrm{FU}$ induced mucositis. Cancer 1996; 77(3):552-5.

36. Al-Refai AS. Immunohistochemical study of the effect of chamomile extract on 5-fluorouracil induced intestinal mucositis in albino rats. J Clin Cell Immunol 2014; 5:1-10.

37. Zadeh JB, Moradi Kor N, Moradi Kor Z. Chamomile (Matricaria recutita as a valuable medicinal plant. IJABBR 2014; 2(3):823-9.

38. Cavalieri E, Rigo A, Bonifacio M, Carcereri de Prati A, Guardalben E, Faculi $M$, et al. Pro- apoptotic activity of $\alpha$-bisabolol in preclinical models of primary human acute leukemia cells. J Transl Med 2011; 9:45-51. 